\title{
A new optimization model for railway slot allocation
}

\author{
B. H. Park ${ }^{1}$, S.-H. Hong ${ }^{2}$ \& C.-S. Kim ${ }^{1}$ \\ ${ }^{1}$ Department of Railroad Management and Logistics, Korea National \\ University of Transportation, Korea \\ ${ }^{2}$ Convergence Technology Research Team, Korea Railroad Research \\ Institute, Korea
}

\begin{abstract}
This study addresses a railway slot allocation model for the case of multiple competing train operating companies (TOC). The auction-based mechanisms have been studied by some researchers, to efficiently allocate the railway slots to TOCs. But the use of such approaches requires the solving of the associated winner determination problem, which can be modelled as a type of timetabling. We consider the profit-maximizing slot allocation problem, where the profit is reduced linearly depending on the deviation from the requested time. Our study suggests a new optimization model based on path-flow variables in a time-space network and a column generation approach using this formulation. The associated subproblem of finding a new path then simply becomes one of finding a shortest path for each requested slot. Compared to other approaches, our approach has the advantage of easily reflecting combinatorial bids like AND or XOR bid. We also present the experimental results applying our approach to a small network.

Keywords: railway slot, column generation, auction.
\end{abstract}

\section{Introduction}

The 2004 functional reform of the Korean railway system was designed to separate the management of railway infrastructure from that of train operations. This resulted in two separate organizations : the infra manager, and train operating company, KORAIL, which runs inter-regional train services that include highspeed trains (KTX). 
Over the last decades, many studies have taken place in Europe regarding open access to railway networks. More recently in Korea, there has been some pressure to increase competition by introducing a second train-operating company (TOC) for the high-speed train service.

The main problem with open access to railway networks is the development of an efficient mechanism of allocating railway slots to each TOC. Several relevant auction-based mechanisms for this have been developed, including Borndörfer et al. [1], Nilsson [2], Brewer and Plott [3], and Parkes and Ungar [4]. These approaches require solving the winner determination problem, which is often formulated as a type of timetabling problem.

Our study addresses a new optimization model for the timetabling problem in the situation where multiple TOCs compete for access to the rail network. This model can be used in auction approaches to slot allocation. We focused on the profit-maximizing slot allocation problem, where the profit is reduced linearly depending on the deviation from the requested times. Such a penalized profit approach has already considered by Caprara et al. [5] and Brännlund et al. [6]. However, our model is based on path-flow variables for easy derivation of the column-generation approach. Unlike previous approaches, our model has the advantage of easily reflecting combinatorial bids like AND or XOR. Even though such combinatorial bids were by Borndörfer et al. [1], their extended integer programming model required inclusion of many binary variables corresponding to such bids.

The remainder of this paper is organized as follows. Section 2 explains the timespace network used in our model. Section 3 presents a new model based on pathflow variables, and the column generation approach, and explains their application to combinatorial bids. Section 4 is devoted to the experimental results of applying our approach to an example network.

\section{Railway slot allocation problem}

\subsection{Time-space network}

Our study is similar to the works [5,6], in that the formulations are based on A network flow model coupled with conflict-free conditions on the set of arcs in the following time-space network.

Each node on a horizontal line represents a discretized time while all the nodes together on any line belong to a single station. An arc between two nodes belonging to different stations represents the travelling times between those two stations. Where nodes corresponding to an intermediate station are split, the arc between them represent the dwell time. To express the time windows of both departure and arrival time, we include two artificial nodes, each of which is connected to its associated principal node within certain acceptable deviation of departure and arrival times.

Most operational constraints can be expressed as packing constraints [1], which means that the number of chosen slots in the conflicting set of arcs should be 


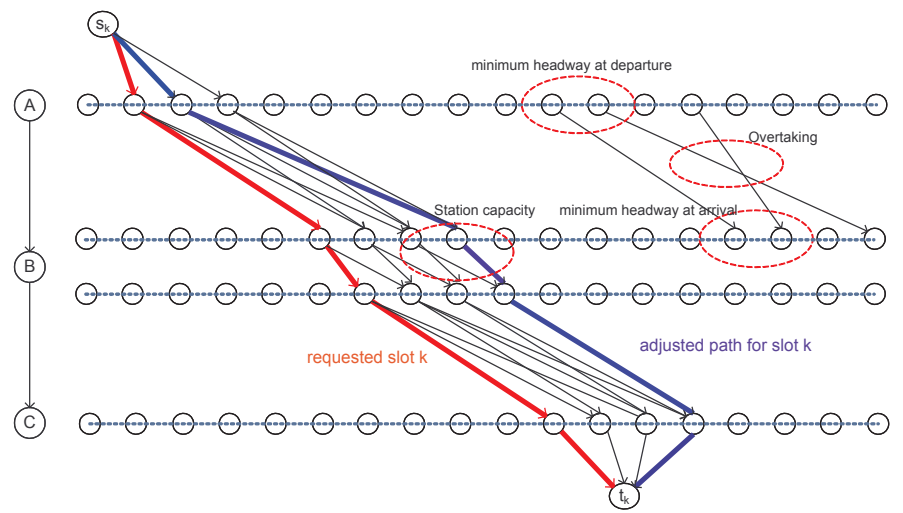

Figure 1: Time-space network, requested and adjusted path for slot $k$, and conflict conditions.

restricted to a constant. As shown in Fig. 1, these constraints can be categorized as one of three types : those for guaranteeing the minimum headway at departure and arrival, those for preventing one train from overtaking another, and those for restricting the number of trains stopped simultaneously at any station.

\subsection{Our problem}

Each TOC submits its set of requested slots, where Slot means the departure time, the arrival time, and the dwell time at each station stop. A slot thus corresponds to the sequence of several arcs in the time-space network, an example of which is indicated by the bold red lines in Fig. 1. A slot can be adjusted within a certain to avoid conflicts. Tolerances can be imposed on the times for travel between stations, or for the departure, arrival, or dwell times at a station. The bold blue lines in Fig. 1 indicate the adjusted path for slot $k$. A slot is accompanied by a bid price. The bid price is adjusted depending on the deviation from the exact slot times.

Our problem is to find adjusted conflict-free paths for the requested slots submitted by the TOCs while maximizing the total profit.

\section{Column generation approach}

\subsection{Formulation}

We used the following notation in constructing our mathematical formulation.

\section{Notation}

- $K:$ the set of requested slots, the elements of which are indexed by $k$

- $V$ : the set of stations in a railway network 
- $A$ : the set of arcs between stations

- $V_{t}$ : the set of nodes in the time-space network the elements of which are indexed by $i, j$

- $A_{t}$ : the set of nodes in the time-space network the elements of which are indexed by $i j$

- $V_{t}^{k}, A_{t}^{k}$ : the set of nodes (arcs) in the time-space network associated with requested slot $k$

- $s_{k}, t_{k}$ : the artificial nodes representing departure (arrival) times of slot $k$

- $n(i) \in V$ : the station index corresponding to node $i \in V_{t}$

- $p_{k}$ : the bid price of slot $k$

- $\rho_{k}^{a r r}$ : the deviation factor associated with the difference between the requested arrival time and the rescheduled arrival time

- $\rho_{k}^{d e p}$ : the deviation factor associated with the difference between the requested departure time and the rescheduled departure time.

- $\operatorname{dep}(k, v), \operatorname{arr}(k, v):$ the requested departure and arrival time of slot $k$ at the station $v$, respectively.

- $\Omega$ : the collection of the sets of conflicting arcs

- $l_{C}:$ the allowed number of arcs in the set $C$

We assume for simplicity that penalties are assigned only to the deviations from the requested departure and arrival times of the two end stations, where the first deviation is the same as the shift defined by [5]. To calculate the penalized profit of the adjusted path for the slot $k$, arc cost $c_{i j}^{k}$ can be assigned to the arcs as follows:

$$
c_{i j}^{k}= \begin{cases}p_{k}-\rho_{k}^{\operatorname{dep}}(j-\operatorname{dep}(k, n(j))) & \text { if } i=s_{k} \\ -\rho_{k}^{\text {arr }}(j-i-\operatorname{arr}(k, n(i))) & \text { if } j=t_{k}\end{cases}
$$

In our study, we present a new formulation based on path flow variables and a column generation algorithm. To do this, we first let $\mathcal{P}_{k}$ as the set of paths from $s_{k}$ to $t_{k}$ on network $G_{k}=\left(V_{t}^{k}, A_{t}^{k}\right)$. We also let $f^{k}(P)$ represent whether the requested slot $k$ uses path $P$ in the time-space network. The formulation, which is called master problem in the context of Dantzig-Wolfe decomposition [7], then becomes the following:

\section{Master problem for slot allocation}

$$
\begin{gathered}
\max \sum_{k \in K} \sum_{P \in \mathcal{P}_{k}} c^{k}(P) f^{k}(P) \\
\text { s.t. } \sum_{P \in \mathcal{P}_{k}} f^{k}(P) \leq 1, \quad \forall k \in K \\
\sum_{k \in K} \sum_{\substack{P \in \mathcal{P}_{k} \mid P \cap C \neq \emptyset \\
f^{k}(P) \in\{0,1\}}} f^{k}(P) \leq l_{C}, \forall C \in \Omega
\end{gathered}
$$


where $c^{k}(P)=\sum_{i j \in P} c_{i j}^{k}$.

The model maximizes the total profit according to eqn. (2). The profit is reduced depending on the deviations from the exact times of the slots. And eqn. (3) indicates that the number of chosen paths should be no greater than 1 for slot $k$. Finally, (4) guarantee a conflict-free timetable.

\subsection{Column-generation approach}

Replacing $f^{k}(P) \in\{0,1\}$ with $f^{k}(P) \geq 0$, we let the nonnegative dual variables $\pi_{k}, \mu_{C}$ correspond to eqn. (3) and eqn. (4), respectively. Thus, the optimality condition of the relaxed master problem is as follows. For all $P \in \mathcal{P}_{k}$,

$$
\begin{gathered}
\bar{c}^{k}(P)=c^{k}(P)-\sum_{\{C \mid P \cap C \neq \emptyset\}} l_{C} \mu_{C}=\sum_{i j \in P} c_{i j}^{k}-\sum_{i j \in P} \sum_{\{C \mid(k, i j) \in C\}} l_{C} \mu_{C}-\pi_{k} \\
=\sum_{i j \in P}\left[c_{i j}^{k}-\sum_{\{C \mid(k, i j) \in C\}} l_{C} \mu_{C}\right]-\pi_{k} \leq 0
\end{gathered}
$$

Thus, the pricing subproblem for slot $k$ is to find a new path $P \in \mathcal{P}_{k}$ such that $\bar{c}^{k}(P)>0$. To find such a path, we assign a modified cost to the $\operatorname{arcs}$ in $A_{t}^{k}$, i.e. $c_{i j}^{k}-\sum_{\{C \mid(k, i j) \in C\}} l_{C} \mu_{C}$. It is then sufficient to find a longest path in the reduced acyclic network, which can be computed rapidly [8]. The overall algorithm is as follows:

\section{Column-generation-based heuristic}

Step 1 Set up the initial restricted master problem comprised of all requested slots.

Step 2 If the termination criterion is satisfied, go to Step 4; otherwise, solve the linear relaxation of the restricted master problem and the pricing problem for all requested slots.

Step 3 If path $P$ exists for the slot $k$ such that $\bar{c}^{k}(P)>0$, then add a new column and a corresponding objective coefficient to the restricted linearly relaxed master problem and go to Step 2.

Step 4 Solve the master problem using the branch-and-bound algorithm.

\subsection{Handling combinatorial bids}

Borndörfer et al. [1] considered combinatorial bids in their extended integer programming model for optimal slot allocation. Combinatorial bids enable TOCs to request slot packages with AND or XOR relationship. Borndörfer et al. used AND bids for the tour, and XOR bids for optional stops. They developed an extended integer program to handle such bids.

In our formulation, however, it is easier to reflect such bids simply by creating an artificial slot. For example, AND and XOR bids of slots $k_{1}$ and $k_{2}$ are denoted by $\operatorname{AND}\left(k_{1}, k_{2}\right)$ and $\operatorname{XOR}\left(k_{1}, k_{2}\right)$, correspond to two new artificial slots, 
from $\mathrm{AND}_{d}\left(k_{1}, k_{2}\right)$ to $\mathrm{AND}_{a}\left(k_{1}, k_{2}\right)$, and from $\mathrm{XOR}_{d}\left(k_{1}, k_{2}\right)$ to $\mathrm{XOR}_{a}\left(k_{1}, k_{2}\right)$, respectively.

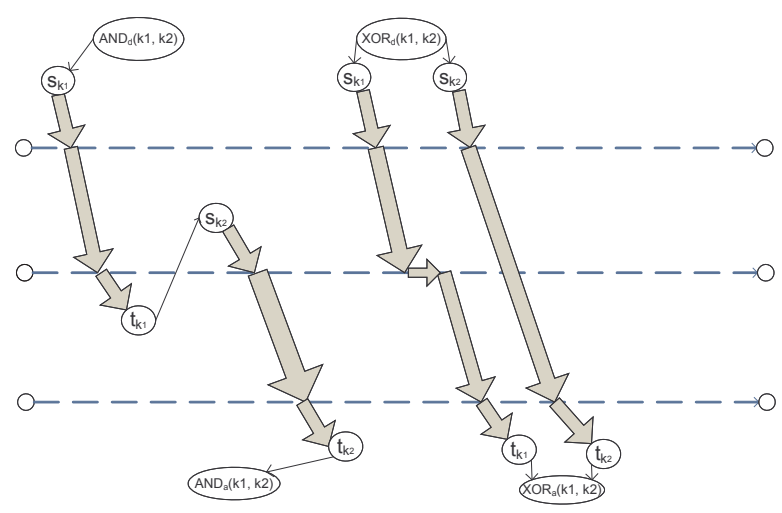

Figure 2: Artificial slots corresponding to AND and XOR bids, for slots $k_{1}, k_{2}$.

\section{Experimental results}

Fig. 3 shows the example railway network used in our experiments. This network is a simplified version of the Korean high-speed railway network. Two TOCs are assumed to be operating trains on routes A-C-D-E, A-C-D-F, B-C-D-E, and B-C-D-F. We generated up to 165 slots by adding delayed copies of the trains with departure time from 05:15 am to 11:55 am in the current timetable. In our experiment, we assumed that all trains had homogeneous rolling-stock so their operating speeds were the same. And we assume that the driving time between two stations is fixed. Therefore there is no need to include the conflict-sets preventing overtaking in $\Omega$.

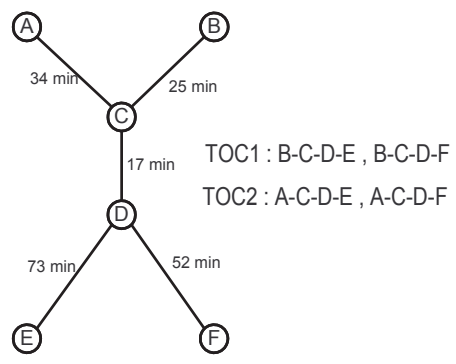

Figure 3: Example network. 
We established tolerances for the dwell times with time window of $[0,5]$ and for departure times from the station of origin with a time window of $[0, d]$. As described in eqn. (1), the deviation penalties were imposed on the departure and arrival times at the two end stations. The bid prices are randomly chosen in the interval between 100 and 110 for all slots and the deviation penalty was set to 1 . Finally, the algorithm terminates if the number of non-increasing iterations appears consecutively no less than 3 . The algorithm was implemented using CPLEX 10.2 on a PC with an Intel Core Duo 2.20-GHz CPU.

In our first experiment, we investigated how well the algorithm works and how rapidly the computation time goes up when the number of requested slots varies. The first column in Table 1 indicates the number of slots which are requested from TOCs, among which the numbers of adjusted slots to be accepted are shown in the third column. The second column indicates the number of chosen slots without any adjustment, i.e. the result of the case that $d=0$. As shown in the fifth column of the table, even in the case that the number of requested slots equals to 165 , it takes just 173 seconds to find an optimal solution.

Table 1: Experimental results 1.

\begin{tabular}{c||c|c|c|c}
\hline \multirow{2}{*}{$\begin{array}{c}\text { \# of requested slots } \\
(=|K|)\end{array}$} & \multicolumn{2}{|c|}{ \# of the accepted slots } & \multirow{2}{*}{ Total profit } & \multirow{2}{*}{ Time (sec.) } \\
\cline { 2 - 3 } & $d=0$ & $d=10$ & & \\
\hline \hline 57 & 42 & 57 & 4,421 & 18 \\
113 & 59 & 87 & 8,718 & 83 \\
165 & 69 & 94 & 9,385 & 173 \\
\hline
\end{tabular}

Table 2: Experimental results 2 - when $|K|=113$.

\begin{tabular}{c||c|c|c}
\hline$d$ & \# of the accepted slots & Total profit & Time (sec.) \\
\hline \hline 0 & 59 & 6,185 & 1 \\
5 & 83 & 8,430 & 24 \\
10 & 87 & 8,718 & 83 \\
\hline
\end{tabular}

Table 2 shows the effect of the time window on the departure time. First, when $d=0$, i.e. when there is no room to adjust, 59 of the 113 slots are selected without any adjustment. However, as $d$ increases, the number of adjusted slots and total penalized profit increases. Interestingly, the computation time does not increase rapidly as $d$ becomes larger.

Even though our results cannot be directly compared with previous works, which considered larger networks and more trains, the results are encouraging in 
that for the case of $d=10$, the total computation time was just 83 seconds while the case of $d=5$ took three days in [1].

\section{Concluding remarks}

We have presented a new formulation and column-generation approach for the railway slot allocation problem. Because our model is just for one round in the framework of iterative auction mechanisms like that of $i$ Bundle [9], it should be coupled with an adequate auction mechanism, which will be our interest in the future research.

\section{References}

[1] Borndörfer, R., Grotschel, M., Lukac, S., Mitusch, K., Schlechte, T., Schultz, S. and Tanner, A., An auctioning approach to railway slot allocation. ZIBReport, 05-45, 2005.

[2] Nilsson, J.-E., Allocation of track capacity: Experimental evidence on the use of priority auctioning in the railway industry. International Journal of Industrial Organization, 17:1139-1162, 1999.

[3] Brewer, P. J. and Plott, C. R., A binary conflict ascending price (BICAP) mechanism for the decentralized allocation of the right to use railroads tracks. International Journal of Industrial Organization, 14:857-886, 1996.

[4] Parkes, D. C. and Ungar, L. H., An auction-based method for decentralized train scheduling. In Proceedings of the Fifth International Conference on Autonomous Agents, 2001.

[5] Caprara, A., Fischetti, M. and Toth, P., Modeling and solving the train timetabling problem. Operations Research, 50(5), 2001.

[6] Brännlund, U., Lindberg, P. O., Nöu, A. and Nilsson, J.-E., Railway timetabling using lagrangian relaxation. Transportation Science, 32(4), 1998.

[7] Wolsey, L. A., Integer Programming. John Wiley and Sons, INC., 1998.

[8] Ahuja, R. K., Magnanti, T. L. and Orlin, J. B. Network Flows: Theory, Algorithms, and Applications. Prentice Hall, New Jersey, 1993.

[9] Parkes, D. C. and Ungar, L. H. ibundle: An efficient ascending price bundle auction. In ACM conference on Electronic Commerce, 1999. 